\title{
Association Between Low-Dose Aspirin and Uric Acid in the Elderly: An Observational Retrospective Cross-Sectional Study
}

\section{Jia-Run Li (D) \\ Yan Fan \\ Mei-Lin Liu}

Department of Geriatrics, Peking University First Hospital, Beijing, 100034 . People's Republic of China
Correspondence: Mei-Lin Liu Tel +86 I391025I863

Email liumeilin@hotmail.com
Purpose: Uric acid is an independent factor for arteriosclerotic cardiovascular disease (ASCVD). Although aspirin is one of the most widely used agent in patients with ASCVD, there were only a few studies focusing on the effects of low-dose aspirin on uric acid metabolism with controversial results. The present study aimed to investigate an association between low-dose aspirin treatment for more than one month and serum uric acid (SUA) with its urinary excretion in elderly patients.

Patients and Methods: This paper presents an observational retrospective cross-sectional study to determine the association between continuous daily taking low-dose aspirin (50$100 \mathrm{mg}$ ) for more than one month and SUA with fraction excretion of uric acid (FEUA) in elderly patients. A total of 506 inpatients equal or over 60 in Department of Geriatrics of Peking University First Hospital were enrolled from 2017 to 2020. About 41.9\% of them were taking aspirin for more than one month, while others were not taking this medicine. The correlation between aspirin use and SUA or FEUA was analyzed, and group-comparison was performed in different dosage groups of aspirin.

Results: After correcting confounding factors, there is no remarkable correlation between taking low-dose aspirin and SUA or FEUA, but a decreasing trend (coefficients=-4.946) of SUA in hyperuricemia patients with low-dose aspirin was observed despite no obvious difference $(\mathrm{P}=0.534)$. Whether SUA or FEUA has no significant difference between $50 \mathrm{mg} /$ $\mathrm{d}$ and $100 \mathrm{mg} / \mathrm{d}$ aspirin subjects.

Conclusion: SUA and urinary uric acid excretion are not associated with using of 50$100 \mathrm{mg} / \mathrm{d}$ aspirin for more than one month in elderly patients with ASCVD or at risk.

Keywords: aspirin, uric acid, excretion, arteriosclerotic cardiovascular disease, elderly

\section{Introduction}

Cardiovascular disease (CVD) is the leading cause of death and disease burden in China and worldwide. ${ }^{1,2}$ Low-dose aspirin is the cornerstone of secondary prevention of ASCVD. However, some researchers discovered that low-dose aspirin could raise SUA by a decrease in uric acid excretion, causing hyperuricemia which is a common clinical condition that can be defined as SUA level $>420 \mathrm{umol} / 1(7 \mathrm{mg} / \mathrm{dL})$ in male or $>360 \mathrm{umol} / 1(6 \mathrm{mg} / \mathrm{dL})$ in female. ${ }^{3}$ Numerous evidence ${ }^{4-6}$ have suggested the existence of an association between elevated SUA and CVD, traditional cardiovascular risk factors, metabolic syndrome, insulin resistance, chronic kidney disease and heart failure. ${ }^{7-9}$ Hyperuricemia is an independent predictor not only of all-cause and cardiovascular mortality, but also of myocardial infarction, stroke 
and heart failure ${ }^{10}$ and it may act as a promoter of renal damage for correlating with lower renal glomerular filtration rate and macroalbuminuria. ${ }^{11}$ But there are few studies focusing on the effects of low-dose aspirin treatment for a longer time (used to be no more than 2 weeks) on uric acid and its urinary excretion among Chinese elderly patients. Therefore, the purpose of the present study was to assess the association between using low-dose aspirin for at least more than one month and serum uric acid with its urinary excretion in elderly patients who combine with ASCVD or have an increasing risk.

\section{Methods}

\section{Study Design and Population}

In this retrospective, cross-sectional and observational study, patients were included if they were equal or more than 60 years old and: (1) have not taken aspirin before or in the last week; (2) taking low-dose aspirin (50-100mg/d) for at least more than one month. And patients were excluded if they were: (1) using drugs that might influence the metabolism of uric acid including diuretics (which could increase SUA with the result of an increased urate renal reabsorption, such as hydrochlorothiazide, loop diuretics and spironolactone, and diuretic-related hyperuricemia may be associated with an adverse cardiovascular outcome $^{12}$ ), uric acid lowering drugs (allopurinol, benzbromarone), some angiotensin receptor blocker (losartan, irbesartan), immunosuppressant agents, SGLT-2 inhibitor, fenofibrate, cytotoxic chemotherapy and anti-tubercular drugs; ${ }^{13}$ (2) having other comorbidities including hypohepatia (Child-Pugh C class), renal insufficiency (eGFR less than $30 \mathrm{~mL} / \mathrm{min}^{*} 1.73 \mathrm{~m}^{2}$ ) or intestinal post-surgery; (3) drinking.

In the overall analysis, studied subjects were divided into groups according to the level of uric acid (Hyperuricemia group and Normal uric acid group) and urinary uric acid excretion (Decreased FEUA group, Normal FEUA group and Increased FEUA group) respectively. Hyperuricemia was defined as SUA>420umol/ $1(7 \mathrm{mg} / \mathrm{dL})$ in male or $>360 \mathrm{umol} / 1(6 \mathrm{mg} / \mathrm{dL})$ in female $^{3}$ while Normal uric acid included other patients. Decreased FEUA was defined as FEUA $<7 \%$ while Increased FEUA was FEUA $>12 \%$ and Normal FEUA was in the middle. ${ }^{14}$ Between-group differences and multivariate regression were performed. Besides, SUA and FEUA were compared among different dosage groups of aspirin.

\section{Clinical Characteristics Collection}

Collected index included sex, age, body mass index (BMI), underlying health conditions, medication, alanine transaminase (ALT), aspartate transaminase (AST), serum creatinine, SUA, estimated glomerular filtration rate (eGFR), systolic blood pressure (SBP), blood lipids including total cholesterol (TC), triglycerides (TG), highdensity lipoprotein cholesterol (HDL-C) and low-density lipoprotein cholesterol (LDL-C), high sensitive C-reaction protein (hsCRP), urinary uric acid, urinary creatinine as well as FEUA which represents the level of urinary uric acid excretion and was calculated by the formula below.

FEUA $^{15}=$ urinary uric acid*serum creatinine/(serum uric acid*urinary creatinine) $* 100 \%$.

\section{Statistical Analysis}

Normally distributed data are expressed as mean \pm SD and were analyzed with independent sample $t$-test or ANOVA test, while non-normally distributed data are presented as median (IQR) and were evaluated by Mann-Whitney $U$-test or Kruskal-Wallis test. Counting data are expressed as a proportion and were determined by Chi-square test. Linear analysis was used to clarify the relativity between aspirin therapy and SUA or FEUA. Statistical significance was taken as $\mathrm{P}<0.05$. All analyses were performed using SPSS version 25.0.

To ensure comparability, we divided participants into three ASCVD risk groups and used the "ASCVD risk" variable to adjust population differences in comorbidities including hypertension, coronary heart disease (CHD), diabetes and hyperlipidemia in multivariate regression analysis. The 2017 Chinese Cardiovascular Disease Prevention Guidelines ${ }^{16}$ were used to estimate 10 -year ASCVD risk (Table 1). Furthermore, our participants were not spread evenly in "sex" or "SUA stratification". We brought these two variables into multivariate regression to reduce selection bias and conducted analyses of the aspirin-SUA association stratified on sex and SUA stratification respectively.

\section{Results}

\section{Baseline Characteristics of the Subjects Descriptive Variables}

Overall, $41.9 \%$ of participants were taking low-dose aspirin while $58.1 \%$ were not using this medicine. The characteristics of descriptive variables in different groups according to aspirin use are summarized in Table 2 and 
Table I 10-Year ASCVD Risk Estimation Flow Chart in Chinese Cardiovascular Disease Prevention Guidelines (2017)

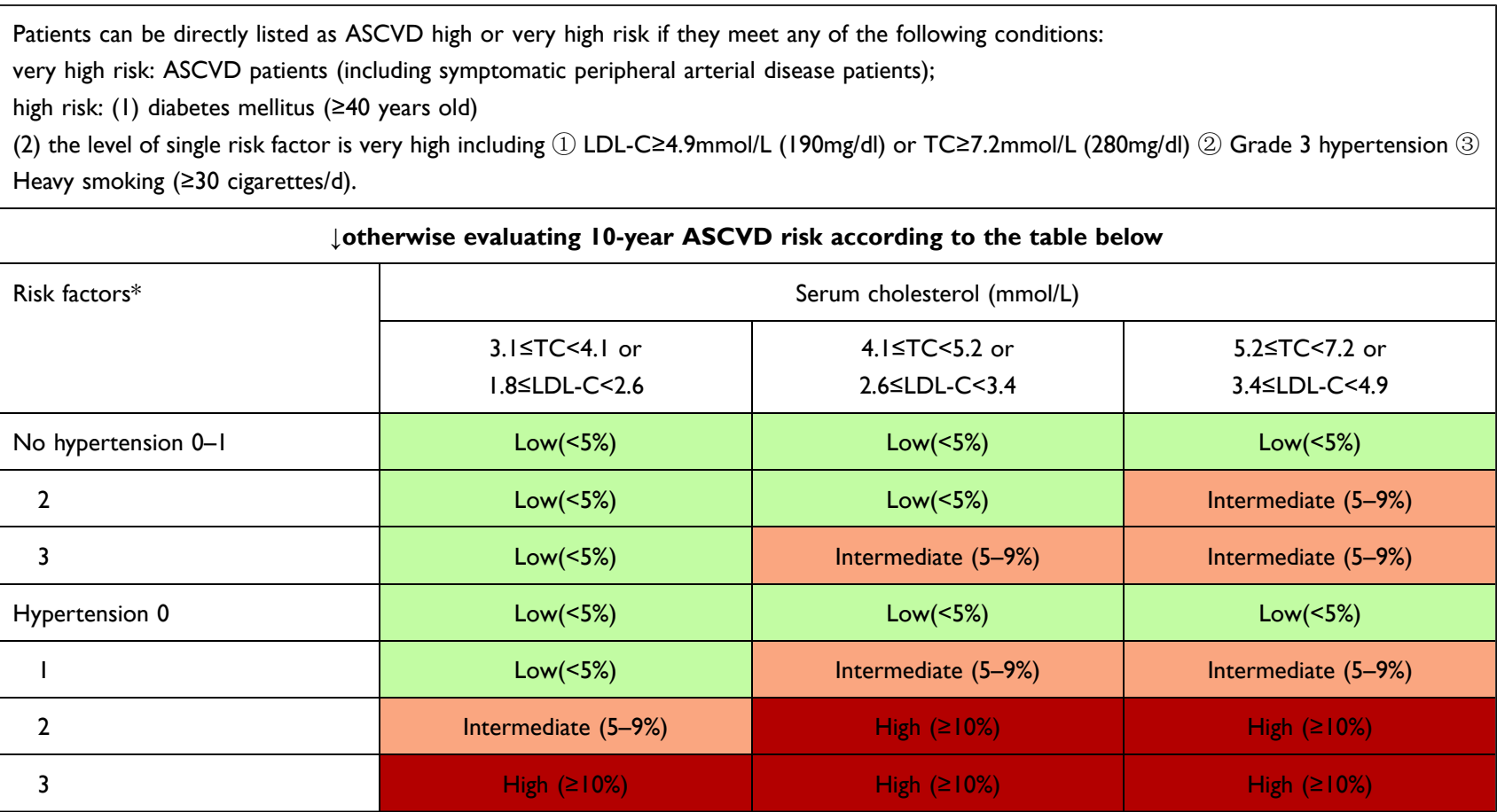

Notes: *including smoking, HDL-C $<1 \mathrm{mmol} / \mathrm{L}$ and age $\geq 45$ years in male or $\geq 55$ years in female. Green highlighting means ASCVD low risk (average risk of disease is $<5 \%$ ); orange highlighting means ASCVD intermediate risk (average risk of disease is 5-9\%); red highlighting means ASCVD high risk (average risk of disease is $\geq 10 \%$ ).

Abbreviations: ASCVD, arteriosclerotic cardiovascular disease; LDL-C, low-density lipoprotein cholesterol; TC, total cholesterol; HDL-C, high-density lipoprotein cholesterol.

Table 2 Baseline Characteristics with Low-Dose Aspirin Use

\begin{tabular}{|c|c|c|c|}
\hline \multirow[t]{2}{*}{ Variables } & \multicolumn{2}{|c|}{ Aspirin Use } & \multirow[t]{2}{*}{$\mathbf{P}$} \\
\hline & No, $n=294$ & Yes, $n=2 \mid 2$ & \\
\hline CHD n(\%) & 51 (I7.3\%) & 152 (7I.7\%) & $<0.00 I^{*}$ \\
\hline Hypertension n(\%) & $173(58.8 \%)$ & I 35 (63.7\%) & 0.271 \\
\hline Diabetes n(\%) & $85(28.9 \%)$ & 91 (42.9\%) & $0.00 I^{*}$ \\
\hline Hyperlipidemia n(\%) & $267(90.8 \%)$ & $212(100 \%)$ & $<0.001 *$ \\
\hline SUA stratification(hyperuricemia) $n(\%)$ & $64(21.8 \%)$ & $36(17.0 \%)$ & 0.182 \\
\hline Decreased FEUA n(\%) & $155(52.7 \%)$ & 117 (55.2\%) & 0.735 \\
\hline Sex(men) $n(\%)$ & $196(66.7 \%)$ & I8I (85.4\%) & $<0.001 *$ \\
\hline Age (years) & $73(17)$ & $76(16)$ & $0.032^{*}$ \\
\hline BMI (kg/m2) & $23.6(4.4)$ & $24.6(4.8)$ & $0.007^{*}$ \\
\hline $\mathrm{SBP}(\mathrm{mmHg})^{\mathrm{a}}$ & $122.0 \pm 11.6$ & $122.5 \pm 12.5$ & 0.680 \\
\hline Glucose $(\mathrm{mmol} / \mathrm{L})^{\mathrm{b}}$ & $5.9(0.7)$ & $6.0(0.9)$ & $0.015^{*}$ \\
\hline $\mathrm{TC}(\mathrm{mmol} / \mathrm{L})^{\#}$ & $4.2(1.3)$ & $3.4(1.0)$ & $<0.00 I^{*}$ \\
\hline TG $(\mathrm{mmol} / \mathrm{L})^{\#}$ & $\mathrm{I} .4(0.8)$ & $1.2(0.9)$ & 0.052 \\
\hline $\mathrm{HDL}-\mathrm{C}(\mathrm{mmol} / \mathrm{L})^{\#}$ & I.I (0.4) & $\mathrm{I} .0(0.3)$ & $0.010^{*}$ \\
\hline LDL-C (mmol/L) $)^{\#}$ & $2.4(1.0)$ & $\mathrm{I} .8(0.7)$ & $<0.001 *$ \\
\hline eGFR $\left(\mathrm{mL} / \mathrm{min} * 1.73 \mathrm{~m}^{2}\right)$ & $77.4(22.0)$ & $74.4(21.3)$ & 0.103 \\
\hline
\end{tabular}

Notes: $* \mathrm{P}<0.05 .{ }^{\mathrm{a}} \mathrm{SBP}$ includes 43 missing values; ${ }^{\mathrm{b}} \mathrm{Glucose}$ includes 20 missing values; ${ }^{\#}$ The variable includes 4 missing values.

Abbreviations: CHD, coronary heart disease; SUA, serum uric acid; FEUA, fraction excretion of uric acid; BMI, body mass index; SBP, systolic blood pressure; TC, total cholesterol; TG, triglycerides; HDL-C, high-density lipoprotein cholesterol; LDL-C, low-density lipoprotein cholesterol; eGFR, estimated glomerular filtration rate. 


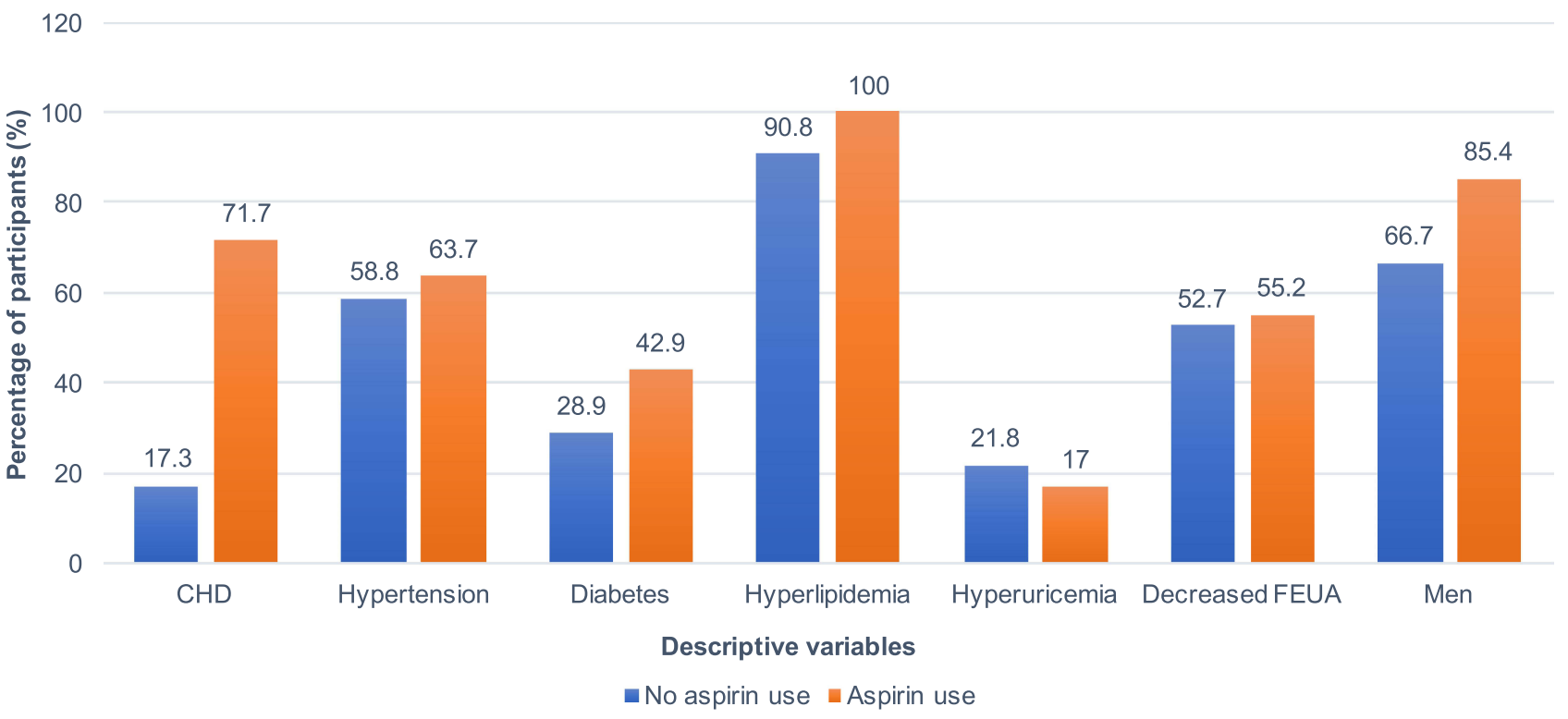

Figure I Distribution of descriptive variables in different aspirin use groups.

Abbreviations: CHD, coronary heart disease; FEUA, fraction excretion of uric acid.

Figure 1. Patients with low-dose aspirin are more likely accompanied with such comorbidities as CHD $(\mathrm{P}<0.001)$, diabetes $(\mathrm{P}=0.001)$ and hyperlipidemia $(\mathrm{P}<0.001)$.

"ASCVD risk" variable was established to revise the population differences in those underlying conditions including hypertension, CHD, diabetes, and hyperlipidemia in subsequent multivariate regression analysis. Following Table 1, participants were divided into ASCVD group ( $\mathrm{n}=205)$, ASCVD low/intermediate-risk group ( $\mathrm{n}=185)$ and high-risk group $(\mathrm{n}=116)$. Also, as Figure 1 showed, our study subjects mostly were male $(66.7 \%$ vs $85.4 \%)$ and a small part of patients was distributed in hyperuricemia $(21.8 \%$ vs $17 \%)$ which may contribute to some research bias. "Sex" and "SUA stratification" variables were brought into subsequent regression analysis.

\section{Continuous Variables}

Collected continuous index including age, BMI, SBP, glucose, TC, TG, HDL-C, LDL-C and eGFR are displayed in Table 2. Patients with low-dose aspirin are more possibly accompanied with older age $(\mathrm{P}=0.032)$, higher $\mathrm{BMI}$ $(\mathrm{P}=0.007)$ and glucose $(\mathrm{P}=0.015)$. Overall levels of blood lipids in patients with aspirin, it should be noted, are better controlled than other subjects and this might be attributed to combination therapy with statins for hyperlipidemia among these patients.

\section{Correlation Factors of SUA}

As shown in Table 3, we compared the baseline characteristics and numbers of using low-dose aspirin patients in two uric acid groups. Of 506 participants included for the analysis, 406 patients were identified as normal uric acid while 100 patients were diagnosed with hyperuricemia. In

Table 3 Univariate Characteristics with SUA

\begin{tabular}{|l|c|c|c|}
\hline \multirow{2}{*}{ Variables } & $\begin{array}{c}\text { Normal } \\
\text { Uric Acid }\end{array}$ & Hyperuricemia & P \\
\cline { 2 - 3 } & $\mathbf{n = 4 0 6}$ & $\mathbf{n}=100$ & \\
\hline CHD n(\%) & $158(38.9 \%)$ & $45(45 \%)$ & 0.266 \\
Hypertension n(\%) & $237(58.4 \%)$ & $71(71 \%)$ & $0.02^{*}$ \\
Diabetes n(\%) & $141(34.7 \%)$ & $35(35 \%)$ & 0.959 \\
Hyperlipidemia n(\%) & $384(94.6 \%)$ & $95(95 \%)$ & 0.867 \\
Aspirin n(\%) & $176(43.3 \%)$ & $36(36 \%)$ & 0.182 \\
Age(years) & $75(16)$ & $74.5(17)$ & 0.692 \\
BMI (kg/m2) & $23.8(4.3)$ & $25.4(4.8)$ & $0.006 *$ \\
Men n(\%) & $302(74.4 \%)$ & $75(75 \%)$ & 0.899 \\
ALT (IU/L) & $16(11)$ & $17(11)$ & 0.316 \\
AST (IU/L) & $22(6)$ & $22(8)$ & 0.494 \\
eGFR(mL/min*I.73m²) & $78.7(21.4)$ & $66.5(20.2)$ & $<0.00 I^{*}$ \\
hsCRP (mg/L) & $0.8(1.2)$ & $1.3(1.7)$ & $<0.00 I^{*}$ \\
HDL-C (mmol/L) & $1.08(0.4)$ & $0.95(0.2)$ & $<0.00 I^{*}$ \\
FEUA (\%) & $7.4(2.8)$ & $5.5(1.6)$ & $<0.00 I^{*}$ \\
\hline
\end{tabular}

Notes: ${ }^{*} \mathrm{P}<0.05$; ${ }^{*} \mathrm{HDL}-\mathrm{C}$ includes 4 missing values.

Abbreviations: CHD, coronary heart disease; BMI, body mass index; ALT, alanine transaminase; AST, aspartate transaminase; eGFR, estimated glomerular filtration rate; hsCRP, high sensitive C-reaction protein; HDL-C, high-density lipoprotein cholesterol; SUA, serum uric acid; FEUA, fraction excretion of uric acid. 
the Hyperuricemia group, more patients have a history of hypertension than those in the Normal uric acid group (71\% vs $58.4 \%, \mathrm{P}=0.02)$. BMI $(\mathrm{P}=0.006)$ and hsCRP $(\mathrm{P}<0.001)$ are higher in the Hyperuricemia group. HDL$\mathrm{C}(\mathrm{P}<0.001)$, eGFR $(\mathrm{P}<0.001)$ and FEUA $(\mathrm{P}<0.001)$ are at a lower level in hyperuricemia patients than normal patients. But the distinction in the frequency of aspirinuse patients between two groups is not obvious ( $43.3 \%$ vs $36 \%, \mathrm{P}=0.182$ ).

By multiple regression analysis, differential variables and other confounding factors including sex, age, ASCVD risk and SUA stratification were selected into the multivariate model. In linear regression as Table 4 shows (adjusted $\mathrm{R}^{2}=0.76$ ), the significant variables are FEUA $(\mathrm{B}=-14.176,95 \% \mathrm{CI}-15.777 \sim-12.575, \mathrm{P}<0.001)$, eGFR $(\mathrm{B}=-1.394, \quad 95 \% \quad \mathrm{CI}-1.697 \sim 1.092, \quad \mathrm{P}<0.001)$, sex (women, $\mathrm{B}=-39.352, \quad 95 \% \quad \mathrm{CI} \quad-47.430 \sim-31.273$, $\mathrm{P}<0.001)$, age $\quad(\mathrm{B}=-0.640,95 \% \quad \mathrm{CI} \quad-1.099 \sim-0.181$, $\mathrm{P}=0.006)$ and $\mathrm{BMI}(\mathrm{B}=1.237,95 \%$ CI $0.147 \sim 2.327$, $\mathrm{P}=0.026$ ). Meanwhile, we did not see obvious effects that low-dose aspirin $(\mathrm{B}=-0.416,95 \%$ CI $-8.265 \sim 7.434$, $\mathrm{P}=0.917$ ) could increase SUA in this model.

The coefficients of sex and SUA stratification are bigger than others as we can see in Table 4 and analyses of aspirin-SUA association according to these two key factors are summarized in Table 5. Either among men or women, taking low-dose aspirin is not associated with SUA, as well as in participants with normal uric acid. Among hyperuricemia patients, however, we could see a decreasing trend of uric acid with using aspirin although the difference is not significant $(\mathrm{B}=-4.946,95 \% \mathrm{CI}$ $-20.683 \sim 10.79, \mathrm{P}=0.534)$.

\section{Relevant Factors of FEUA}

Based on the level of FEUA, participants were divided into Decreased FEUA, Normal FEUA and Increased FEUA groups, including 272, 217 and 17 participants, respectively, as Table 6 lists. Decreased FEUA is significantly correlated with male $(\mathrm{P}<0.001)$, younger age $(\mathrm{P}=0.001)$, higher BMI $(\mathrm{P}<0.001)$ and lower HDL-C $(\mathrm{P}<0.001)$. The odds of aspirin-use patients have no difference among these groups (43\% vs $40.1 \%$ vs $47.1 \%$, $\mathrm{P}=0.735$ ). Likewise, differential variables and confounding factors including ASCVD risk and SUA stratification were chosen into multivariate regression. In linear model as Table 7 displays (adjusted $\mathrm{R}^{2}=0.202$ ), sex (women, $\mathrm{B}=1.111,95 \%$ CI $0.678 \sim 1.545, \mathrm{P}<0.001)$, eGFR $(\mathrm{B}=$ $-0.027,95 \% \mathrm{CI}-0.044 \sim-0.011, \mathrm{P}=0.001)$ and $\mathrm{BMI}(\mathrm{B}=$ $-0.084,95 \% \mathrm{CI}-0.143 \sim-0.024, \mathrm{P}=0.006$ ) are deep relevant factors of FEUA. Aspirin treatment has no significant correlation with urinary uric acid excretion $(\mathrm{B}=0.078,95 \%$ CI $-0.353 \sim 0.509, \mathrm{P}=0.722$ ).

\section{Level of SUA and FEUA in Patients with Different Aspirin Dosage}

Study subjects were grouped according to aspirin dosage and further analyses are on display in Table 8 . There were 187 patients taking $100 \mathrm{mg} / \mathrm{d}$ aspirin while 20 patients with $50 \mathrm{mg} / \mathrm{d}$ aspirin. And 294 patients were not prescribed with treatment of low-dose aspirin. Five patients whose dosage was between $50 \mathrm{mg}$ and $100 \mathrm{mg}$ daily were not brought into statistics because of small quantity and little practicability in clinic. The results show that no significant difference on the level of SUA or FEUA was observed in these groups and indicate that neither $50 \mathrm{mg} / \mathrm{d}$ nor $100 \mathrm{mg} / \mathrm{d}$ aspirin has

Table 4 Linear Regression of SUA

\begin{tabular}{|l|c|c|c|c|c|c|}
\hline Variables & $\mathbf{B}^{\mathbf{\Lambda}}$ & $\mathbf{P}$ & \multicolumn{2}{|c|}{$95 \%$ CI for B } & Tolerance & VIF \\
\hline (constant) & 597.245 & $0.000^{*}$ & 532.693 & 661.797 & & \\
Women & -39.352 & $0.000^{*}$ & -47.430 & -31.273 & 0.869 & \\
Age & -0.640 & $0.006 *$ & -1.099 & $-0.18 \mathrm{I}$ & 0.598 & 1.151 \\
ASCVD risk & 1.096 & 0.629 & -3.362 & 5.555 & 0.704 & 1.672 \\
eGFR & -1.394 & $0.000^{*}$ & -1.697 & -1.092 & 0.575 & 1.420 \\
hsCRP & 0.168 & 0.145 & -0.058 & 0.394 & 0.961 & 1.738 \\
BMI & 1.237 & $0.026 *$ & 0.147 & 2.327 & 0.900 & 1.041 \\
SUA stratification & 91.253 & $0.000^{*}$ & 81.746 & 100.761 & 0.751 & \\
FEUA & -14.176 & $0.000^{*}$ & -15.777 & -12.575 & 0.787 & 1.331 \\
Aspirin & -0.416 & 0.917 & -8.265 & 7.434 & 0.718 & 1.270 \\
\hline
\end{tabular}

Notes: ${ }^{*} \mathrm{P}<0.05 .{ }^{\Delta} \mathrm{B}$ represents unstandardized coefficients.

Abbreviations: VIF, variance inflation factor; ASCVD, arteriosclerotic cardiovascular disease; eGFR, estimated glomerular filtration rate; hsCRP, high sensitive C-reaction protein; BMI, body mass index; SUA, serum uric acid; FEUA, fraction excretion of uric acid. 
Table 5 Different Linear Models of SUA According to Sex and SUA Stratification

\begin{tabular}{|l|c|c|c|c|c|c|}
\hline Model & $\mathbf{R}^{\mathbf{2}}$ & Adjusted $\mathbf{R}^{\mathbf{2}}$ & $\mathbf{B}^{\mathbf{\Delta}}$ (Aspirin) & \multicolumn{2}{|c|}{ 95\% Cl for B } & P \\
\hline Total patients & 0.765 & 0.760 & -0.416 & -8.265 & 7.434 & 0.917 \\
Male & 0.730 & 0.724 & 0.527 & -8.933 & 9.988 & 0.913 \\
Female & 0.787 & 0.772 & 0.662 & -13.369 & 14.694 & 0.926 \\
Normal uric acid & 0.558 & 0.549 & 0.598 & -8.47 & 9.665 & 0.897 \\
Hyperuricemia & 0.510 & 0.466 & -4.946 & -20.683 & 10.79 & 0.534 \\
\hline
\end{tabular}

Note: ${ }^{\Delta} \mathrm{B}$ represents unstandardized coefficients.

Abbreviation: SUA, serum uric acid.

Table 6 Univariate Characteristics with FEUA

\begin{tabular}{|c|c|c|c|c|}
\hline \multirow[t]{2}{*}{ Variables } & Decreased FEUA & Normal FEUA & Increased FEUA & \multirow[t]{2}{*}{$\mathbf{P}$} \\
\hline & $n=272$ & $n=217$ & $n=17$ & \\
\hline CHD n(\%) & 112 (4I.2\%) & 85 (39.2\%) & $6(35.3 \%)$ & 0.830 \\
\hline Hypertension n(\%) & $165(60.7 \%)$ & $134(61.8 \%)$ & $9(52.9 \%)$ & 0.769 \\
\hline Diabetes n(\%) & 87 (32\%) & $82(37.8 \%)$ & 7 (4I.2\%) & 0.348 \\
\hline Hyperlipidemia $n(\%)$ & $259(95.2 \%)$ & 203 (93.5\%) & 17 (100\%) & 0.436 \\
\hline Aspirin n(\%) & 117 (43\%) & 87 (40.1\%) & $8(47.1 \%)$ & 0.735 \\
\hline Age (years) & $73(17)$ & 77 (I5) & $80(16)$ & $0.001 *$ \\
\hline BMI $(\mathrm{kg} / \mathrm{m} 2)$ & $24.8(4.4)$ & $23.4(3.9)$ & $23.2(4.5)$ & $<0.001 *$ \\
\hline Men n(\%) & $223(82 \%)$ & 143 (65.9\%) & II (64.7\%) & $<0.001 *$ \\
\hline ALT (IU/L) & $16(11)$ & $16(11)$ & $15(8)$ & 0.488 \\
\hline AST (IU/L) & $21.5(7)$ & $22(7)$ & $22(10)$ & 0.844 \\
\hline $\operatorname{eGFR}\left(\mathrm{mL} / \mathrm{min}^{*} 1.73 \mathrm{~m}^{2}\right)$ & $77.4(20.8)$ & $72.2(21.3)$ & $79.5(24.4)$ & $0.026 *$ \\
\hline $\mathrm{HDL}-\mathrm{C}(\mathrm{mmol} / \mathrm{L})^{\#}$ & $0.99(0.3)$ & I.I (0.4) & I.I (0.3) & $<0.00 I^{*}$ \\
\hline
\end{tabular}

Notes: ${ }^{*} \mathrm{P}<0.05$. ${ }^{\#} \mathrm{HDL}-\mathrm{C}$ includes 4 missing values.

Abbreviations: CHD, coronary heart disease; BMI, body mass index; ALT, alanine transaminase; AST, aspartate transaminase; eGFR, estimated glomerular filtration rate; HDL-C, high-density lipoprotein cholesterol; FEUA, fraction excretion of uric acid.

Table 7 Liner Regression of FEUA

\begin{tabular}{|l|c|c|c|c|c|c|}
\hline Variables & $\mathbf{B}^{\mathbf{\Lambda}}$ & $\mathbf{P}$ & \multicolumn{2}{|c|}{$95 \%$ CI for B } & Tolerance & VIF \\
\hline (Constant) & 8.757 & $0.000^{*}$ & 5.295 & 12.219 & & \\
Women & $1.1 \mathrm{II}$ & $0.000^{*}$ & 0.678 & 1.545 & 0.913 & 1.095 \\
Age & 0.020 & 0.122 & -0.005 & 0.045 & 0.611 & 1.637 \\
ASCVD risk & -0.026 & 0.832 & -0.271 & 0.219 & 0.705 & 1.418 \\
eGFR & -0.027 & $0.001 *$ & -0.044 & -0.011 & 0.588 & 1.701 \\
BMI & -0.084 & $0.006^{*}$ & -0.143 & -0.024 & 0.915 & 1.093 \\
SUA stratification & -2.056 & $0.000^{*}$ & -2.546 & -1.566 & 0.854 & 1.171 \\
Aspirin & 0.078 & 0.722 & -0.353 & 0.509 & 0.720 & 1.390 \\
\hline
\end{tabular}

Notes: $* \mathrm{P}<0.05 .{ }^{\Delta} \mathrm{B}$ represents unstandardized coefficients.

Abbreviations: VIF, variance inflation factor; ASCVD, arteriosclerotic cardiovascular disease; eGFR, estimated glomerular filtration rate; BMI, body mass index; SUA, serum uric acid; FEUA, fraction excretion of uric acid.

an outstanding correlation with uric acid metabolism in people aged 60 years or older.

\section{Discussion}

Low-dose aspirin has become one of the most popular agents used for the treatment and prevention of ASCVD, especially myocardial infarction and ischemic stroke. The various low-dose aspirin protocols have been extensively studied for their efficacy but not for their possible influence on serum uric acid and urate handling. It is worth to figure out whether low-dose aspirin therapy during a long period could influence SUA or urinary uric acid excretion 
Table 8 SUA and FEUA Among Different Dosage Groups of Aspirin

\begin{tabular}{|l|l|l|l|l|}
\hline \multirow{2}{*}{ Variables } & \multicolumn{2}{|l|}{ Aspirin Use } & \multirow{2}{*}{ No Use } & \multirow{2}{*}{ P } \\
\cline { 2 - 3 } & $\mathbf{1 0 0 \mathrm { mg } / \mathbf { d }}$ & $\mathbf{5 0 \mathrm { mg } / \mathbf { d }}$ & $\mathbf{n}=\mathbf{2 9 4}$ & \\
\cline { 2 - 3 } & $\mathbf{n = 1 8 7}$ & $\mathbf{n = 2 0}$ & & \\
\hline SUA & $349.0 \pm 72.9$ & $354.95 \pm 64.2$ & $341.4 \pm 79.1$ & 0.467 \\
FEUA & $6.9(2.9)$ & $6.2(3.2)$ & $6.8(2.8)$ & 0.763 \\
\hline
\end{tabular}

Abbreviations: SUA, serum uric acid; FEUA, fraction excretion of uric acid.

for guiding a better treatment and management of elderly patients combined with metabolic syndrome.

Uric acid is known to induce endothelial dysfunction by reactive oxygen production ${ }^{17}$ and inflammation ${ }^{18}$ by various mechanisms and relates to CVD. Early researchers thought that low-dose aspirin was associated with hyperuricemia and gout attacks. ${ }^{19}$ However, the effects and potential mechanisms have remained uncertain. Caspi et $\mathrm{al}^{20}$ enrolled 49 elderly inpatients (age 61-94) and found that the week-1 dosage of $75 \mathrm{mg} / \mathrm{d}$ aspirin caused a $15 \%$ decrease in uric acid excretion and an increase in SUA. Nevertheless, Akinwusi et $\mathrm{al}^{21}$ showed that aspirin treatment $(75 \mathrm{mg} / \mathrm{d})$ for two weeks with 4-week follow-up did not affect SUA levels in 30 elderly patients. With more participants included, Zhang et $\mathrm{al}^{22}$ analyzed 446 subjects and gave a conclusion that low-dose aspirin treatment $(50 \mathrm{mg} / \mathrm{d}, 100 \mathrm{mg} / \mathrm{d})$ for two weeks showed no hyperuricemia effect in people over 60 The average creatinine clearance rate (Ccr) in Caspi's study was $47 \mathrm{~mL} / \mathrm{min}$ which was lower than Akinwusi's $(108 \mathrm{~mL} /$ $\mathrm{min})$, Zhang P's $(79 \mathrm{~mL} / \mathrm{min})$ and ours' $(75 \mathrm{~mL} / \mathrm{min})$. It is speculated that the different effects of aspirin on SUA may be related to different renal functions. Our study demonstrates that the treatment of low-dose aspirin $(50-100 \mathrm{mg} / \mathrm{d})$ for more than one month is not associated with SUA. A decreasing trend of uric acid in hyperuricemia patients with low-dose aspirin is observed in this study, although the statistics difference is not significant. Further prospective research including a bigger sample size of hyperuricemia participants is needed to confirm.

The kidney plays an important role in the elimination of uric acid. Little is known, however, about the possible renal effects and mechanisms on uric acid handling of lowdose aspirin. Segal et al $^{23,24}$ observed 83 stable geriatric patients treated with $100 \mathrm{mg} / \mathrm{d}$ aspirin for 2 weeks and found that urinary excretion of uric acid decreased in mostly aspirin group patients with SUA increased. However, the study of Akinwusi et $\mathrm{al}^{21}$ did not show any deleterious effects with low-dose $(75 \mathrm{mg} / \mathrm{d})$ aspirin use on kidney functions in 30 elderly patients during 6 weeks. Ali et $\mathrm{al}^{25}$ found that $75 \mathrm{mg} / \mathrm{d}$ aspirin had no significant effect on renal allograft function over the 15-year study period post-transplant among 147 patients (mean age 44.5). Goicoechea et $\mathrm{al}^{26}$ assessed 116 patients with CKD 3-4 stage and found long-term (mean follow-up time $64.8 \pm$ 16.4 months) aspirin treatment $(100 \mathrm{mg} / \mathrm{d})$ may be a protective factor of renal disease progression. In the past two decades, researchers have sought to determine low-dose aspirin how to effect metabolism of uric acid. Some researchers found that low-dose aspirin was able to inhibit MRP4-mediated urate secretion in human embryonic kidney cells ${ }^{27}$ which may attribute to less uric acid excretion in urine. On the contrary, Massimi et $\mathrm{al}^{28}$ reported that aspirin caused overexpression of MRP4. Other investigators thought that low-dose aspirin reduced thromboxane ${ }^{29}$ and may ameliorate the progression of renal impairment, ${ }^{30}$ improving glomerular circulation with more uric acid excreted. Selective thromboxane inhibition also improved renal function in lupus nephritis and renal prostacyclin production was preserved. ${ }^{31}$ The complicated mechanisms of aspirin involved in renal function and urate handling have not been explained completely. It is indicated that the increased level of uric acid with decreased urinary excretion of uric acid observed among aspirin users in previous studies are more likely because of small quantity and short-term observation.

However, far too little attention has been paid to lowdose aspirin on relatively large number of elderly during a longer period. To our best knowledge, this is the largest study to explore the association between low-dose aspirin (50-100mg/d) using for more than one month and SUA with its urinary excretion among elderly patients. In this study, 506 patients over 60 were enrolled and results imply that low-dose aspirin treatment for more than one month is not associated with SUA or urinary uric acid excretion. This is a crucial finding in the understanding of the impacts of low-dose aspirin on uric acid metabolism. And in our study, group-comparison in Table 8 brings us to an important point that the dosage whether $50 \mathrm{mg}$ or $100 \mathrm{mg}$ daily of low-dose aspirin is not related to uric acid or its excretion.

More than a century after its synthesis, daily aspirin, given at a low dose, is a milestone treatment for the secondary prevention of ASCVD. Its role in primary prevention is still debated. Making clinical decision regarding the aspirin use for primary prevention of ASCVD events is still complex. Many adults were not using aspirin appropriately $^{32}$ considering risk on bleeding or metabolic 
disorder, though evidence for the efficacy and safety of aspirin for the primary prevention of ASCVD has been accumulating since $1988 .{ }^{33}$ Our study provides the first demonstration that there is no significant association between taking low-dose aspirin for more than one month and SUA or urinary uric acid excretion. And we suggest it is not necessary to be overly cautious that lowdose aspirin used for primary prevention of ASCVD may increase SUA or decrease urinary excretion of uric acid if the medicine is prescribed for more than one month.

The limitations of this study as follows: This is a single-center, retrospective, descriptive study, which limits the generalizability of the observations. The sample size of $50 \mathrm{mg} / \mathrm{d}$ aspirin subjects is small so that subgroup analysis maybe lack accuracy. More studies need to be down to figure out the effects of this dosage on uric acid metabolism, considering the latent bleeding risk of $100 \mathrm{mg} /$ d for elderly patients with cerebrovascular or gastrointestinal diseases. Due to practical constraints, this study cannot provide a long-time observation. Further research focusing on the impact of a longer-term like 3-5 years use of low-dose aspirin on uric acid and its metabolism is still essential.

\section{Conclusion}

In summary, the daily use of $50-100 \mathrm{mg}$ aspirin for more than one month is not associated with serum uric acid or its urinary excretion despite the dosage in elderly patients with ASCVD or at risk. SUA correlates with variables including FEUA, eGFR, sex, age and BMI, while the urinary excretion of uric acid, FEUA, is related to eGFR, sex and BMI. For hyperuricemia subjects, in our study, SUA possibly has a slight decrease after using low-dose aspirin for more than one month which demands more research about hyperuricemia patients with aspirin treatment to make it clear.

\section{Data Sharing Statement}

The data generated or analyzed during the current study are available from the corresponding author on reasonable request.

\section{Ethics Approval and Informed Consent}

All procedures performed in studies involving human participants were in accordance with the 1964 Helsinki Declaration and its later amendments or comparable ethical standards. This study was approved by the Research Ethics Committee of Peking University First Hospital and approval number is 2020 scientific research 105. Informed consent of this study has been granted an exemption by this committee because all information was collected from the medical record system and is maintained with confidentiality. This is a retrospective study and there is no need to collect additional blood samples, schedule extra inspections, conduct further medical interventions or to follow up.

\section{Acknowledgments}

We appreciate the Medical Statistics Room of Peking University First Hospital for their invaluable assistance with data analysis.

\section{Author Contributions}

All authors contributed to data analysis, drafting or revising the article, have agreed on the journal to which the article will be submitted, gave final approval of the version to be published, and agree to be accountable for all aspects of the work.

\section{Funding}

This study was funded by the National Key Research and Development Project of China (2016YFC1301300, 2016YFC1301304).

\section{Disclosure}

The authors declare that they have no conflict of interest.

\section{References}

1. Zhou M, Wang H, Zhu J, et al. Cause-specific mortality for 240 causes in China during 1990-2013: a systematic subnational analysis for the Global Burden of Disease Study 2013. Lancet. 2016;387(100 15):251-272.

2. Mortality GBD. Causes of Death C. Global, regional, and national age-sex specific all-cause and cause-specific mortality for 240 causes of death, 1990-2013: a systematic analysis for the Global Burden of Disease Study 2013. Lancet. 2015;385(9963):117-171.

3. Multidisciplinary Expert Task Force on H, Related D. Chinese multidisciplinary expert consensus on the diagnosis and treatment of hyperuricemia and related diseases. Chin Med $J$ (Engl). 2017;130 (20):2473-2488.

4. Li M, Hu X, Fan Y, et al. Hyperuricemia and the risk for coronary heart disease morbidity and mortality a systematic review and dose-response meta-analysis. Sci Rep. 2016;6:19520.

5. Fang J, Alderman MH. Serum uric acid and cardiovascular mortality the NHANES I epidemiologic follow-up study, 1971-1992. National Health and Nutrition Examination Survey. JAMA. 2000;283 (18):2404-2410.

6. Holme I, Aastveit AH, Hammar N, Jungner I, Walldius G. Uric acid and risk of myocardial infarction, stroke and congestive heart failure in 417,734 men and women in the Apolipoprotein MOrtality RISk study (AMORIS). J Intern Med. 2009;266(6):558-570. 
7. Ndrepepa G. Uric acid and cardiovascular disease. Clin Chim Acta. 2018;484:150-163.

8. Srivastava A, Kaze AD, McMullan CJ, Isakova T, Waikar SS. Uric acid and the risks of kidney failure and death in individuals with CKD. Am J Kidney Dis. 2018;71(3):362-370.

9. Borghi C, Rosei EA, Bardin T, et al. Serum uric acid and the risk of cardiovascular and renal disease. J Hypertens. 2015;33(9):17291741; discussion 1741.

10. Maloberti A, Giannattasio C, Bombelli M, et al. Hyperuricemia and risk of cardiovascular outcomes: the experience of the URRAH (Uric Acid Right for Heart Health) Project. High Blood Press Cardiovasc Prev. 2020;27(2):121-128.

11. Russo E, Viazzi F, Pontremoli R, et al. Association of uric acid with kidney function and albuminuria: the Uric Acid Right for heArt Health (URRAH) Project. J Nephrol. 2021.

12. Maloberti A, Bombelli M, Facchetti R, et al. Relationships between diuretic-related hyperuricemia and cardiovascular events: data from the URic acid Right for heArt Health study. J Hypertens. 2021;39 (2):333-340.

13. Ben Salem C, Slim R, Fathallah N, Hmouda H. Drug-induced hyperuricaemia and gout. Rheumatology (Oxford). 2017;56(5):679-688.

14. Ra T. Primer on the rheumatic diseases.11th ed. Atlanta: Arthritis Foundation. Epidemiol Pathol Pathogenesis. 1997;230-243.

15. Li M, Li X, Liu Y, et al. Relationship between serum bilirubin levels $\mathrm{S}$ and the progression of renal function in patients with chronic kidney disease and hyperuricemia. Clin Chim Acta. 2018;48 6:156-161.

16. Task Force on Chinese Guidelines for the Prevention of Cardiovascular D, Editorial Board of Chinese Journal of C. [Chinese guidelines for the prevention of cardiovascular diseases (2017)]. Zhonghua Xin Xue Guan Bing Za Zhi. 2018;46(1):10-25.

17. Maruhashi T, Hisatome I, Kihara Y, Higashi Y. Hyperuricemia and endothelial function: from molecular background to clinical perspectives. Atherosclerosis. 2018;278:226-231.

18. Muhammad Mansyur Romi NA, Tranggono U, Wahyu Setyaningsih WA, Ratna Sari DC. Uric acid causes kidney injury through inducing fibroblast expansion, Endothelin-1 expression, and inflammation. BMC Nephrol. 2017;18(1):326.

19. Zhang Y, Neogi T, Chen C, Chaisson C, Hunter DJ, Choi H. Lowdose aspirin use and recurrent gout attacks. Ann Rheum Dis. 2014;73 (2):385-390.

20. Caspi D, Lubart E, Graff E, Habot B, Yaron M, Segal R. The effect of mini-dose aspirin on renal function and uric acid handling in elderly patients. Arthritis Rheum. 2000;43(1):103-108.
21. Akinwusi PO, Oluyombo R, Ogunro PS, Adeniji AO, Okunola OO, Ayodele OE. Low dose aspirin therapy and renal function in elderly patients. Int J Gen Med. 2013;6:19-24.

22. Zhang P, Wang H, Chen XH, Liang WY, Liu WW, Liu ML. Effect of low-dose aspirin on serum uric acid levels in Chinese individuals over 60: subanalysis of a multicentre randomized clinical trial. Eur Rev Med Pharmacol Sci. 2020;24(5):2719-2724.

23. Segal R, Lubart E, Leibovitz A, et al. Early and late effects of low-dose aspirin on renal function in elderly patients. Am J Med. 2003;115(6):462-466.

24. Segal R, Lubart E, Leibovitz A, Iaina A, Caspi D. Renal effects of low dose aspirin in elderly patients. Isr Med Assoc J. 2006;8 (10):679-682.

25. Ali H, Shaaban A, Murtaza A, Howell LE, Ahmed A. Effect of long-term, low-dose aspirin therapy on renal graft function. Exp Clin Transplant. 2017;15(4):400-404.

26. Goicoechea M, de Vinuesa SG, Quiroga B, et al. Aspirin for primary prevention of cardiovascular disease and renal disease progression in chronic kidney disease patients: a multicenter randomized clinical trial (AASER Study). Cardiovasc Drugs Ther. 2018;32(3):255-263.

27. El-Sheikh AA, van den Heuvel JJ, Koenderink JB, Russel FG. Effect of hypouricaemic and hyperuricaemic drugs on the renal urate efflux transporter, multidrug resistance protein 4. Br J Pharmacol. 2008;155 (7):1066-1075.

28. Massimi I, Ciuffetta A, Temperilli F, et al. Multidrug resistance Protein-4 influences aspirin toxicity in human cell line. Mediators Inflamm. 2015;2015:607957.

29. Pedersen AK, FitzGerald GA. Dose-related kinetics of aspirin. Presystemic acetylation of platelet cyclooxygenase. $N$ Engl J Med. 1984;311(19):1206-1211.

30. Purkerson ML, Joist JH, Yates J, Valdes A, Morrison A, Klahr S. Inhibition of thromboxane synthesis ameliorates the progressive kidney disease of rats with subtotal renal ablation. Proc Natl Acad Sci US A. 1985;82(1):193-197.

31. Pierucci A, Simonetti BM, Pecci G, et al. Improvement of renal function with selective thromboxane antagonism in lupus nephritis. N Engl J Med. 1989;320(7):421-425.

32. Luepker RV, Oldenburg NC, Misialek JR, et al. Aspirin use and misuse for the primary prevention of cardiovascular diseases. $\mathrm{Am}$ J Prev Med. 2021.

33. Patrono $\mathrm{C}$, Baigent $\mathrm{C}$. Role of aspirin in primary prevention of cardiovascular disease. Nat Rev Cardiol. 2019;16(11):675-686.
International Journal of General Medicine

\section{Publish your work in this journal}

The International Journal of General Medicine is an international, peer-reviewed open-access journal that focuses on general and internal medicine, pathogenesis, epidemiology, diagnosis, monitoring and treatment protocols. The journal is characterized by the rapid reporting of reviews, original research and clinical studies across all disease areas. The manuscript management system is completely online and includes a very quick and fair peer-review system, which is all easy to use. Visit http://www.dovepress.com/ testimonials.php to read real quotes from published authors. 